\title{
Consistency and the sequential equal contributions rule for airport problems
}

Citation for published version (APA):

Chun, Y., Kayi, C., \& Yeh, C-H. (2008). Consistency and the sequential equal contributions rule for airport problems. METEOR, Maastricht University School of Business and Economics. METEOR Research Memorandum No. 039 https://doi.org/10.26481/umamet.2008039

Document status and date:

Published: 01/01/2008

DOI:

10.26481/umamet.2008039

Document Version:

Publisher's PDF, also known as Version of record

\section{Please check the document version of this publication:}

- A submitted manuscript is the version of the article upon submission and before peer-review. There can be important differences between the submitted version and the official published version of record.

People interested in the research are advised to contact the author for the final version of the publication, or visit the DOI to the publisher's website.

- The final author version and the galley proof are versions of the publication after peer review.

- The final published version features the final layout of the paper including the volume, issue and page numbers.

Link to publication

\footnotetext{
General rights rights.

- You may freely distribute the URL identifying the publication in the public portal. please follow below link for the End User Agreement:

www.umlib.nl/taverne-license

Take down policy

If you believe that this document breaches copyright please contact us at:

repository@maastrichtuniversity.nl

providing details and we will investigate your claim.
}

Copyright and moral rights for the publications made accessible in the public portal are retained by the authors and/or other copyright owners and it is a condition of accessing publications that users recognise and abide by the legal requirements associated with these

- Users may download and print one copy of any publication from the public portal for the purpose of private study or research.

- You may not further distribute the material or use it for any profit-making activity or commercial gain

If the publication is distributed under the terms of Article $25 \mathrm{fa}$ of the Dutch Copyright Act, indicated by the "Taverne" license above, 
Youngsub Chun, Çağatay Kay1, Chun-Hsien Yeh

Consistency and the sequential equal

contributions rule for airport problems

$\mathrm{RM} / 08 / 039$

JEL code: C71, D30, D63

\section{METE@R}

Maastricht research school of Economics of TEchnology and ORganizations

Universiteit Maastricht

Faculty of Economics and Business Administration P.O. Box 616

NL - 6200 MD Maastricht

phone : ++31433883830

fax $\quad:++31433884873$ 


\section{Consistency and the sequential equal contributions rule for airport problems*}

\author{
Youngsub Chun ${ }^{\dagger}$ \\ School of Economics \\ Seoul National University \\ Seoul 151-746, Korea
}

\author{
Çă̆atay Kayı \\ Department of Economics \\ Maastricht University \\ P.O. Box 6166200 MD \\ Maastricht, The Netherlands
}

\author{
Chun-Hsien Yeh ${ }^{\S}$ \\ Institute of Economics \\ Academia Sinica \\ Taipei 115, Taiwan \\ Department of Economics \\ National Central University \\ Chung-Li 320, Taiwan
}

November 6, 2008

${ }^{*}$ We would like to thank Bettina Klaus and William Thomson for their helpful and detailed suggestions and discussions.

$\dagger$ E-mail: ychun@snu.ac.kr

${ }^{\ddagger}$ E-mail: C.Kayi@algec.unimaas.nl

${ }^{\S}$ Corresponding author; Tel.: +886-2-2782-2791 ext 511; Fax: +886-2-2785-3946; Email: chyeh@econ.sinica.edu.tw ; chyeh@mgt.ncu.edu.tw (C.-H. Yeh). 


\begin{abstract}
We consider the problem of sharing the cost of a public facility among agents who have different needs for it. We base two characterizations of the sequential equal contributions rule on smallest-cost consistency. Namely, (i) the rule is the only rule satisfying equal treatment of equals, independence of all but the smallest-cost, and smallest-cost consistency, and (ii) it is the only rule satisfying equal share lower bound, cost monotonicity, and smallest-cost consistency. Journal of Economics Literature Classification Numbers: C71; D30; D63.
\end{abstract}

Keywords: consistency; sequential equal contributions rule; airport problems; TU games; the Shapley value.

\title{
1 Introduction
}

We consider a class of cost sharing problems with the following features: agents are ordered in terms of their needs for a public facility, and satisfying a given agent implies satisfying all agents with smaller needs than his. An example is the so-called "airport problem": different airlines need airstrips of different lengths. The larger a plane, the longer the airstrip it needs. Serving a given plane implies serving all smaller planes. To accommodate all planes, the airstrip must be long enough for the largest plane. How should the cost of the airstrip be shared among the airlines? ${ }^{1}$ A "rule" is a function that associates with each airport problem, an allocation of the cost of the airstrip, called a "contribution vector". A number of properties of rules have been formulated for this problem and are motivated by various fairness principles. The literature devoted to the search for rules satisfying these properties, singly and in various combinations, is initiated by Littlechild and Owen (1973). ${ }^{2}$

\footnotetext{
${ }^{1}$ Another application is the so-called "irrigation problems": ranchers are distributed along an irrigation ditch. A rancher only needs the part of the ditch from his field to the headgate. To accommodate all ranchers, the ditch should reach the furthest field from the headgate. How should the cost of maintaining the ditch be shared among the ranchers?

${ }^{2}$ For a comprehensive survey of this literature, see Thomson (2005).
} 
A well-known rule is the "sequential equal contributions" (SEC) rule, which has been employed in real world for many years. ${ }^{3}$ To introduce the rule, suppose that there are $n$ airlines indexed by $1, \ldots, n$. Let $l_{i}$ be the length of airstrip that airline $i$ needs, and $c_{i}$ the cost parameter of airline $i$ (the cost of building such airstrip that airline $i$ needs). For simplicity, assume that $l_{1}<\cdots<l_{n}$, and that the cost parameter is a strictly increasing function of airstrip length. Thus, $c_{1}<\cdots<c_{n}$. Imagine that the airstrip is composed of "segments": the first segment is defined by $l_{1}$, the second segment $l_{2}-l_{1}$, the third segment $l_{3}-l_{2}$, and so on. In addition, the cost of the first segment is $c_{1}$, the cost of the second segment $c_{2}-c_{1}$, the cost of the third segment $c_{3}-c_{2}$, and so on. All airlines using a given segment contribute equally to the cost of the segment, and thus pay the total of the contributions of each segment that they use. (Note that Littlechild and Owen (1973) show that the contributions vector recommended by the SEC rule coincides with that prescribed by the "Shapley value" (Shapley, 1953) applied to the TU game associated to the problem in a natural way. ${ }^{4}$ )

Our purpose here is to base axiomatic characterizations of the SEC rule on the following variable-population property, which is an application for airport problems of a general principle of "consistency" ${ }^{6}$. Consider a contributions vector $x$ chosen by a rule for the problem just defined. Imagine that airline 1

\footnotetext{
${ }^{3}$ The terminology we adopt is borrowed from Thomson (2005). This rule has been discussed by Baker and Associates (1965) and Littlechild and Owen (1973). It underlies the "serial" idea that has been the subject of a number of studies by many authors. For instance, Moulin and Shenker (1992). Aadland and Kolpin (1998) refer to it as the serial cost-share rule, and explain that it is standard in allocating the cost of irrigation ditch in south-central Montana.

${ }^{4}$ Given an airport problem, we first transform the problem into a TU game, which we call the associated airport game, by defining the worth of each coalition as the cost of building the airstrip used by an airline with the largest cost parameter in that coalition. We then apply a TU game solution to solve the game. This yields a payoff vector. Finally, we take this payoff vector as the contributions vector for the airport problem.

${ }^{5}$ For a survey of the literature on consistency and its converse, see Thomson (2000).

${ }^{6} \mathrm{~A}$ number of authors have provided several axiomatic characterizations of the SEC rule. Readers are referred to Dubey (1982), Moulin and Shenker (1992), Aadland and Kolpin (1998), and Potters and Sudhölter (1999).
} 
pays his contribution $x_{1}$ and "leaves", and reassess the situation from the viewpoint of the remaining airlines. It is of course natural to think of $x_{1}$ as a contribution to the part of the airstrip that airline 1 uses. Since contributing to the part of the airstrip that airline 1 uses implies contributing to the part of the airstrip that all other airlines use, the cost parameters of the remaining airlines are then revised down by the amount $x_{1}$. "Smallest-cost consistency" of the rule requires that for the reduced problem just defined, each of the remaining airlines should contribute the same amount as he did initially. ${ }^{7}$

In addition to smallest-cost consistency, we consider the following desirable properties. The first property is a symmetry property, "equal treatment of equals": two airlines with the same cost parameters should contribute equal amounts. The second property is an independence property, "independence of all but the smallest-cost": if the cost parameters of all airlines other than an airline with the smallest cost parameter increase by the same positive amount, the airline with the smallest cost parameter should contribute the same amount as he did initially. The third one is a lower bound requirement on each airline's contribution. Imagine, for each airline separately, that his cost parameter is the smallest. We then divide his cost parameter by the numbers of airlines. (Note that any segment the airline uses is jointly used by all other airline. Thus, an equal share of the cost parameter of the airline is very natural.) "Equal share lower bound" of the rule requires that each airline should contribute at least as much as an equal share of his cost parameter. The last one is a monotonicity property, "cost monotonicity": if an airline's cost parameter increases, all other airlines should contribute at most as much as they did initially.

\footnotetext{
${ }^{7}$ When another airline leaves, it is not easy to define the reduced problem. Potters and Sudhölter (1999) propose two types of consistency requirements for airport problems, " $\nu$ consistency" and " $\psi$-consistency". Depending on which formation of a reduced problem is adopted, we are led to different consistency properties. However, when we focus on the departure of an airline with the smallest cost parameter, $\nu$-consistency and $\psi$-consistency coincide with smallest-cost consistency. Compared to $\nu$-consistency and $\psi$-consistency, smallest-cost consistency is natural and no controversial at all.
} 
Our contribution is that we offer two consistency characterizations of the SEC rule. Namely, (i) the rule is the only rule satisfying equal treatment of equals, independence of all but the smallest-cost, and smallest-cost consistency, and (ii) it is the only rule satisfying equal share lower bound, cost monotonicity, and smallest-cost consistency.

The rest of this paper proceeds as follows. Section 2 introduces the model, the nucleolus, and the properties. Section 3 present the main result and show the independence of the properties.

\section{Notation and definitions}

There is a universe of "potential" agents, denoted by $\mathcal{I} \subseteq \mathbb{N}$ where $\mathbb{N}$ is the set of natural numbers. Let $\mathcal{N}$ be the class of non-empty and finite subsets of $\mathcal{I}$. Given $N \in \mathcal{N}$ and $i \in N$, let $c_{i} \in \mathbb{R}_{+}$be agent $i$ 's cost parameter, and $c \equiv\left(c_{i}\right)_{i \in N}$ the profile of cost parameters. An airport problem for $N$, or simply a problem for $\boldsymbol{N}$, is a list $c \in \mathbb{R}_{+}^{N}$. Let $\mathcal{C}^{N}$ be the class of all problems for $N$. A contributions vector for $c \in \mathcal{C}^{N}$ is a vector $x \in \mathbb{R}^{N}$ such that it is "efficient", i.e., $\sum_{i \in N} x_{i}=\max _{i \in N} c_{i}$ and "reasonable", i.e., for each $i \in N, 0 \leq x_{i} \leq c_{i}$. Let $X(c)$ be the set of all contributions vectors for $c \in \mathcal{C}^{N}$. A rule is a function defined on $\bigcup_{N \in \mathcal{N}} \mathcal{C}^{N}$ that associates with each $N \in \mathcal{N}$ and each $c \in \mathcal{C}^{N}$ a vector in $X(c)$. Let $n$ denote the number of agents in $N$ and $\eta:\{1, \ldots, n\} \rightarrow N$ be a bijection such that $c_{\eta(1)} \leq \cdots \leq c_{\eta(n)}$. Thus, the agents in $N$ are ordered in terms of their cost parameters. Note that if several agents have the same cost parameters, then the order is not unique. For convention, we assume that $N \equiv\{1, \ldots, n\}$ and $c_{1} \leq \cdots \leq c_{n}$. Our generic notation for rules is $S$. For each coalition $N^{\prime} \subset N$, we denote $\left(c_{i}\right)_{i \in N^{\prime}}$ by $c_{N^{\prime}},\left(S_{i}(c)\right)_{i \in N^{\prime}}$ by $S_{N^{\prime}}(c)$, and so on. All terminologies we adopt are borrowed from Thomson (2005)

We now formally introduce the sequential equal contributions rule.

Sequential equal contributions rule, SEC: For each $N \in \mathcal{N}$, each $c \in$ 
$\mathcal{C}^{N}$, and each $i \in N$,

$$
S E C_{i}(c) \equiv \frac{c_{1}}{n}+\frac{c_{2}-c_{1}}{n-1}+\cdots+\frac{c_{i}-c_{i-1}}{n-i+1} .
$$

The SEC rule satisfies the following properties informally defined in the introduction.

Equal treatment of equals: For each $N \in \mathcal{N}$, each $c \in \mathcal{C}^{N}$, and each pair $\{i, j\} \subseteq N$, if $c_{i}=c_{j}$, then $S_{i}(c)=S_{j}(c)$.

Equal share lower bound: For each $N \in \mathcal{N}$, each $c \in \mathcal{C}^{N}$, and each $i \in N$, $S_{i}(c) \geq \frac{c_{i}}{n}$.

Independence of all but the smallest-cost: For each $N \in \mathcal{N}$, each $c \in \mathcal{C}^{N}$, each $c^{\prime} \in \mathcal{C}^{N}$, and each $\delta \geq 0$, if $c_{1}^{\prime}=c_{1}$ and for each $i \in N \backslash\{1\}$, $c_{i}^{\prime}=c_{i}+\delta$, then $S_{1}\left(c^{\prime}\right)=S_{1}(c)$.

Cost monotonicity: For each $N \in \mathcal{N}$, each $c \in \mathcal{C}^{N}$, each $c^{\prime} \in \mathcal{C}^{N}$, and $i \in N$, if $c_{i}^{\prime} \geq c_{i}$ and for each $j \in N \backslash\{i\}, c_{j}^{\prime}=c_{j}$, then for each $j \in N \backslash\{i\}$, $S_{j}\left(c^{\prime}\right) \leq S_{j}(c){ }^{8}$

Next is the central property to our analysis. Let $N \in \mathcal{N}, c \in \mathcal{C}^{N}, x \in$ $X(c)$, and $i^{*} \in\left\{i \in N \mid\right.$ for each $\left.k \in N, c_{i} \leq c_{k}\right\}$. The reduced problem of $\boldsymbol{c}$ with respect to $\boldsymbol{N}^{\prime} \equiv \boldsymbol{N} \backslash\left\{\boldsymbol{i}^{*}\right\}$ and $\boldsymbol{x}, r_{N^{\prime}}^{x}(c)$, is defined by setting for each $j \in N^{\prime}$,

$$
\left(r_{N^{\prime}}^{x}(c)\right)_{j} \equiv c_{j}-x_{i^{*}}
$$

Smallest-cost consistency: For each $N \in \mathcal{N}$, each $c \in \mathcal{C}^{N}$, and each $N^{\prime} \subset N$, if $x \equiv S(c)$, then $r_{N^{\prime}}^{x}(c) \in \mathcal{C}^{N^{\prime}}$ and $x_{N^{\prime}}=S\left(r_{N^{\prime}}^{x}(c)\right)$.

Remark: Reasonableness and smallest-cost consistency together imply efficiency.

\footnotetext{
${ }^{8}$ This property is introduced by Thomson (2005). The property is a complement of "individual cost monotonicity" (Potters and Sudhölter, 1999), which says the following. Under the same hypotheses, agent $i$ should pay at least as much as he did initially.
} 


\section{The results}

Our first result is that the SEC rule is the only rule satisfying equal treatment of equals, independence of all but the smallest-cost, and smallest-cost consistency. To prove this assertion, we use the fact that the SEC rule satisfies the following monotonicity property: if the cost parameters of all agents increase by the same positive amount, each agent should contribute at least as much as he did initially.

Uniform-cost-increase monotonicity: For each $N \in \mathcal{N}$, each $c \in \mathcal{C}^{N}$, each $c^{\prime} \in \mathcal{C}^{N}$, and each $\delta>0$, if for each $i \in N, c_{i}^{\prime}=c_{i}+\delta$, then for each $i \in N, S_{i}(c) \leq S_{i}\left(c^{\prime}\right)$.

Note that the SEC rule satisfies uniform-cost-increase monotonicity is an immediate consequence of the definition of the SEC rule. Thus, the proof of this fact is omitted. We are now ready to prove the announced assertion.

Theorem 1 The SEC rule is the only rule satisfying equal treatment of equals, independence of all but the smallest-cost, and smallest-cost consistency.

Proof. Clearly, the SEC rule satisfies equal treatment of equals, independence of all but the smallest-cost, and smallest-cost consistency. Conversely, let $S$ be a rule satisfying the properties. Let $N \in \mathcal{N}$ and $c \in \mathcal{C}^{N}$. Let $x \equiv S(c)$ and $y \equiv S E C(c)$. We show that $x=y$. The proof is by induction on $n$.

Case 1: $\mathbf{n}=1$. By efficiency of the rule, $x=y$.

Case 2: $\mathbf{n}>1$. The induction hypothesis is that for each $N^{\prime} \in \mathcal{N}$ and $c^{*} \in$ $\mathcal{C}^{N^{\prime}}$ with $N^{\prime} \subset N$ and $\left|N^{\prime}\right| \leq n-1$, we have $S\left(c^{*}\right)=S E C\left(c^{*}\right)$. We first show that $x_{1}=y_{1}$. By smallest-cost consistency and the induction hypothesis, we then conclude that $x=y$. So, let $N^{\prime}=N \backslash\{1\}$. We distinguish two cases.

Subcase 2.1: $\mathbf{c}_{\mathbf{1}}=\mathbf{c}_{\mathbf{2}}$. Suppose, by contradiction, that $x_{1} \neq y_{1}$. Thus, either $x_{1}>y_{1}$ or $x_{1}<y_{1}$. If $x_{1}>y_{1}$, then by equal treatment of equals, $x_{2}>y_{2}$. 
By smallest-cost consistency, $x_{2}=S_{2}\left(r_{N^{\prime}}^{x}(c)\right)$ and $y_{2}=S E C_{2}\left(r_{N^{\prime}}^{y}(c)\right)$. Since $x_{1}>y_{1}$, then for each $i \in N^{\prime},\left(r_{N^{\prime}}^{x}(c)\right)_{i} \leq\left(r_{N^{\prime}}^{y}(c)\right)_{i}$. Note that $\left|N^{\prime}\right|<n$. By the induction hypothesis, $S_{2}\left(r_{N^{\prime}}^{x}(c)\right)=S E C_{2}\left(r_{N^{\prime}}^{x}(c)\right)$. Since for each $i \in N^{\prime}$, $\left(r_{N^{\prime}}^{y}(c)\right)_{i}-\left(r_{N^{\prime}}^{x}(c)\right)_{i}=x_{1}-y_{1}>0$, thus the two reduced problems $r_{N^{\prime}}^{x}(c)$ and $r_{N^{\prime}}^{y}(c)$ satisfy the hypotheses of uniform-cost-increase monotonicity. Since the SEC rule satisfies uniform-cost-increase monotonicity, it follows that $x_{2} \leq y_{2}$, in violation of $x_{2}>y_{2}$. If $x_{1}<y_{1}$, then by a similar argument, we derive the desired contradiction.

Subcase 2.2: $\mathbf{c}_{\mathbf{1}}<\mathbf{c}_{\mathbf{2}}$. Let $c^{\prime}$ be such that $c_{1}^{\prime}=c_{1}$ and for each $i \in N^{\prime}$, $c_{i}^{\prime} \equiv c_{i}-\left(c_{2}-c_{1}\right)$. Let $x^{\prime} \equiv S\left(c^{\prime}\right)$ and $y^{\prime} \equiv S E C\left(c^{\prime}\right)$. Note that $c_{1}^{\prime}=c_{2}^{\prime}$. By Subcase 2.1, $x_{1}^{\prime}=y_{1}^{\prime}$. By independence of all but the smallest-cost, $x_{1}^{\prime}=x_{1}$ and $y_{1}^{\prime}=y_{1}$. Thus, $x_{1}=y_{1}$.

Q.E.D.

Yeh and Hwang (2007) show that another well-known rule, the "nucleolus", is the only rule satisfying equal treat of equals, "largest-cost additivity" 9 , and "largest-cost consistency" 10 . Their result shows that the nucleolus can be characterized on the basis of properties that almost entirely focus on an agent with the largest cost parameter. Thus, their result reveals the interest of focusing an agent with largest cost parameter in characterizing the nucleolus. In contrast, our Theorem 1 shows that the SEC rule can be characterized on the basis of properties that almost entirely focus on an agent with the smallest cost parameter. Thus, Theorem 1 reveals the interest of focusing on an agent with the smallest cost parameter in characterizing the SEC rule.

We next present another characterization of the SEC rule on the basis of smallest-cost consistency.

\footnotetext{
${ }^{9}$ This property says that if the cost parameter of an agent with the largest cost parameter increases by some positive amount, then the contribution of the agent should increase by the same amount.

${ }^{10}$ This property is a weaker version of $\nu$ consistency (Potters and Sudhölter (1999)). It is obtained by restricting attention to the departure of an agent with the largest cost parameter.
} 
Theorem 2 The SEC rule is the only rule satisfying equal share lower bound, cost monotonicity, and smallest-cost consistency.

Proof. Clearly, the SEC rule satisfies equal share lower bound, cost monotonicity, and smallest-cost consistency. Conversely, let $S$ be a rule satisfying the three properties. Let $x \equiv S(c)$ and $y \equiv S E C(c)$. We show that $x=y$. The proof is by induction on $n$.

Case 1: $\mathbf{n}=\mathbf{1}$. By efficiency of the rule, $x=y$.

Case 2: $\mathbf{n}>1$. The induction hypothesis is that for each $\left(N^{\prime}, c^{*}\right)$ such that $N^{\prime} \subset N$ and $\left|N^{\prime}\right| \leq n-1$, we have $S\left(c^{*}\right)=S E C\left(c^{*}\right)$. We first show that $x_{1}=y_{1}$. By smallest-cost consistency and the induction hypothesis, we then conclude that $x=y$. By equal share lower bound, $x_{1} \geq \frac{c_{1}}{n}$. Suppose that $x_{1}>\frac{c_{1}}{n}$. Let $\bar{c} \in \mathcal{C}^{N}$ be such that for each $i \in N \backslash\{1\}, \bar{c}_{i}=c_{1}$. Let $\bar{x} \equiv S(\bar{c})$. By equal share lower bound and efficiency, $\bar{x}_{1}=\frac{c_{1}}{n}$. Now, let $\bar{c}^{n} \in \mathcal{C}^{N}$ be such that $\bar{c}_{n}^{n}=c_{n}$ and for each $i \in N \backslash\{n\}, \bar{c}_{i}^{n}=\bar{c}_{i}$. Let $\bar{x}^{n} \equiv S\left(\bar{c}^{n}\right)$. By cost monotonicity, $\bar{x}_{1}^{n} \leq \bar{x}_{1}$. Let $\bar{c}^{n-1} \in \mathcal{C}^{N}$ be such that $\bar{c}_{n-1}^{n-1}=c_{n-1}$ and for each $i \in N \backslash\{n-1\}, \bar{c}_{i}^{n-1}=\bar{c}_{i}^{n}$. Let $\bar{x}^{n-1} \equiv S\left(\bar{c}^{n-1}\right)$. By cost monotonicity, $\bar{x}_{1}^{n-1} \leq \bar{x}_{1}^{n}$. Continuing this process, we have $\bar{c}^{2} \equiv c$ and $\bar{x}_{1}^{2} \leq \bar{x}_{1}^{3}$. Since $x=\bar{x}^{2}$, we have $x_{1}=\bar{x}_{1}^{2} \leq \bar{x}_{1}^{3} \leq \cdots \leq \bar{x}_{1}^{n}=\frac{c_{1}}{n}$, which contradicts to the assumption of $x_{1}>\frac{c_{1}}{n}$. Thus, $x_{1}=\frac{c_{1}}{n}$.

We now show that the properties listed in Theorems 1 and 2 are logically independent. For that purpose, we introduce the following rules. The first rule is the "constrained egalitarian rule" (Aadland and Kolpin, 1998). ${ }^{11}$ Start by requiring equal contributions from all agents in $N$ until there are $\gamma^{1} \in \mathbb{R}_{+}$ and $k^{1} \in N$ such that $k^{1} \gamma^{1}=c_{k^{1}}$ (if there are several such $k^{1}$, select the

\footnotetext{
${ }^{11}$ Aadland and Kolpin (1998) name this rule as the restricted average cost-share rule. The authors show that the contributions vector the rule chooses coincides with that prescribed by the "egalitarian rule" (Dutta and Ray, 1989) applied to the associated airport game.
} 
largest). Then, each $i \in\left\{1, \ldots, k^{1}\right\}$ pays $\gamma^{1}$. Continue by requiring equal contributions from members of $\left\{k^{1}+1, \ldots, n\right\}$ until there are $\gamma^{2} \in \mathbb{R}_{+}$and $k^{2} \in N$ such that $k^{1} \gamma^{1}+\left(k^{2}-k^{1}\right) \gamma^{2}=c_{k^{2}}$ (if there are several such $k^{2}$, select the largest). Then each $i \in\left\{k^{1}+1, \ldots, k^{2}\right\}$ pays $\gamma^{2}$. Continue in this way until the total amount collected is $c_{n}$. This algorithm can be expressed as follows.

Constrained Egalitarian rule, $\boldsymbol{C E}$ : For each $N \in \mathcal{N}$ and each $c \in \mathcal{C}^{N}$,

$$
\begin{aligned}
& C E_{1}(c) \equiv \min \left\{\frac{c_{1}}{1}, \cdots, \frac{c_{n}}{n}\right\} \\
& C E_{i}(c) \equiv \min \left\{\frac{c_{k}-\sum_{p=1}^{i-1} C E_{p}(c)}{k-i+1} \mid i \leq k \leq n\right\} \quad 2 \leq i \leq n-1 \\
& C E_{n}(c) \equiv c_{n}-\sum_{p=1}^{n-1} C E_{p}(c) .
\end{aligned}
$$

The second rule is a "weighted version" of the SEC rule. Given $i \in \mathcal{I}$, let $\alpha_{i} \in \mathbb{R}_{++}$be agent $i$ 's weight. Let $N \equiv\{1, \ldots, n\}$ and $\alpha \equiv\left(\alpha_{i}\right)_{i \in N}$ be the weights vector. Without loss of generality, assume that $c_{1} \leq \cdots \leq c_{n}$. Then, the weighted SEC rule with respect to the weights vector $\alpha$ is defined as follows: let $c_{0} \equiv 0$.

Weighted sequential equal contributions rule, $\boldsymbol{S E} \boldsymbol{C}^{\alpha}$ : For each $N \in$ $\mathcal{N}$, each $c \in \mathcal{C}^{N}$, and each $i \in N$,

$$
S E C_{i}^{\alpha}(c) \equiv \frac{\alpha_{i}}{\sum_{j=1}^{n} \alpha_{j}} c_{1}+\frac{\alpha_{i}}{\sum_{j=2}^{n} \alpha_{j}}\left(c_{2}-c_{1}\right)+\cdots+\frac{\alpha_{i}}{\sum_{j=i}^{n} \alpha_{j}}\left(c_{i}-c_{i-1}\right) .
$$

The next rule is a modified version of the SEC rule. When there are three agents and their cost parameters differ, the rule assigns each agent an equal share of the smallest cost parameter plus the difference between his cost parameter and the cost parameter of his immediate predecessor; otherwise, the rule assigns agents the contributions made by the SEC rule.

Modified sequential equal contributions rule, $\boldsymbol{S E} \boldsymbol{C}^{*}$ : For each $N \in \mathcal{N}$, each $c \in \mathcal{C}^{N}$, and each $i \in N$,

$$
S E C_{i}^{*}(c) \equiv\left\{\begin{array}{cl}
S_{i}(c) & \text { if }|N|=3 \text { and } c_{1}<c_{2}<c_{3} \\
S E C_{i}(c) & \text { otherwise }
\end{array}\right.
$$


where $S$ is defined as follows: Let $N \equiv\{1,2,3\}$.

$$
\begin{aligned}
& S_{1}(c)=\frac{c_{1}}{3} \\
& S_{2}(c)=\frac{c_{1}}{3}+c_{2}-c_{1} \\
& S_{3}(c)=\frac{c_{1}}{3}+c_{3}-c_{2}
\end{aligned}
$$

The last rule assigns each agent an equal share of his cost parameter, and then one of the agents with the largest cost parameter pays the remaining amount to be collected. Let $i^{*} \in\left\{i \in N \mid\right.$ for each $\left.k \in N, c_{i} \geq c_{k}\right\}$.

$\boldsymbol{S}^{*}$ : For each $N \in \mathcal{N}$, each $c \in \mathcal{C}^{N}$, and each $i \in N$,

$$
S_{i}^{*}(c) \equiv\left\{\begin{array}{cl}
\frac{c_{i}}{n} & \text { if } i \in N \backslash\left\{i^{*}\right\} ; \\
c_{i^{*}}-\sum_{j \in N \backslash\left\{i^{*}\right\}} \frac{c_{j}}{n} & \text { otherwise, }
\end{array}\right.
$$

\begin{tabular}{|l|c|c|c|c|c|}
\hline Property/Rule & $C E$ & $S E C^{\alpha}$ & $S E C^{*}$ & $S^{*}$ & $S E C$ \\
\hline Equal treatment of equals & + & - & + & - & + \\
\hline Independence of all but the smallest-cost & - & + & + & + & + \\
\hline Smallest-cost consistency & + & + & - & - & + \\
\hline Equal share lower bound & + & - & - & + & + \\
\hline Cost monotonicity & - & + & - & + & + \\
\hline
\end{tabular}

Table 1: Independence of the properties in Theorems 1 and 2 . The notation "+" ("-") means that a certain rule satisfies (violates) a certain property.

Table 1 shows that the properties listed in Theorems 1 and 2 are independent. For instance, the $C E$ rule satisfies equal treatment of equals and smallest-cost consistency but violates independence of all but the smallestcost. The $S E C^{\alpha}$ rule satisfies independence of all but the smallest-cost and 
smallest-cost consistency but violates equal treatment of equals. The $S E C^{*}$ rule satisfies equal treatment of equals and independence of all but the smallestcost but violates smallest-cost consistency. Thus, the properties listed in Theorem 1 are logically independent.

\section{References}

Aadland D., Kolpin V. (1998) "Shared irrigation costs: An empirical and axiomatic analysis", Mathematical Social Sciences 35, 203-218.

Baker and Associates (1965) "Runway cost impact studies", Report presented to the Association of Local Transport Airlines, Jackson, Miss.

Dubey P. (1982) "The Shapley value as aircraft landing fees-revisited", Management Science 28, 869-874.

Dutta B., Ray D. (1989) "A concept of egalitarian under participation constraints", Econometrica 57, 615-635.

Littlechild S.C., Owen G. (1973) "A simple expression for the Shapley value in a special case", Management Science 3, 370-372.

Moulin H., Shenker S. (1992) "Serial cost sharing", Econometrica 60, 10091037.

Potters J., Sudhölter P. (1999) "Airport problems and consistent allocation rules", Mathematical Social Sciences 38, 83-102.

Shapley L.S. (1953) "A value for $n$-person games", in H. Kuhn and A.W. Tucker (eds.), Contributions to the Theory of Games, Vol. 2, 307-317.

Thomson W. (2000) "Consistency and its converse", mimeo. University of Rochester, Rochester, NY, USA.

Thomson W. (2005) "Cost allocation problems and airport problems", mimeo. University of Rochester, Rochester, NY, USA.

Yeh C.-H., Hwang Yan-An (2007) "An alternative characterization of the nucleolus in airport problems", mimeo. Institute of Economics, Academia Sinica, Taipei, Taiwan. 